\title{
A collocation spectral method for two-dimensional Sobolev equations
}

Shiju Jin ${ }^{1}$ and Zhendong Luo ${ }^{2^{*}}$

"Correspondence: zhdluo@163.com ${ }^{2}$ School of Mathematics and Physics, North China Electric Power University, Beijing, China

Full list of author information is available at the end of the article

\begin{abstract}
This article mainly studies a collocation spectral method for two-dimensional (2D) Sobolev equations. To this end, a collocation spectral model based on the Chebyshev polynomials for the 2D Sobolev equations is first established. And then, the existence, uniqueness, stability, and convergence of the collocation spectral numerical solutions are discussed. Finally, some numerical experiments are provided to verify the corrections of theoretical results. This implies that the collocation spectral model is very effective for solving the 2D Sobolev equations.
\end{abstract}

MSC: $65 \mathrm{~N} 30 ; 65 \mathrm{~N} 12 ; 65 \mathrm{M} 15$

Keywords: Collocation spectral method; Sobolev equation; Existence and stability as well as convergence; Numerical experiment

\section{Introduction}

Because any bounded closed domain $\bar{\Omega}$ in $\mathbb{R}^{2}$ can be approximately filled with several rectangles $\left[a_{i}, b_{i}\right] \times\left[c_{i}, d_{i}\right](i=1,2, \ldots, I)$, for convenience and without losing universality, let us just assume that $\bar{\Omega}=[a, b] \times[c, d] \subset \mathbb{R}^{2}$, whose boundary is denoted by $\partial \Omega$, consider the following two-dimensional (2D) Sobolev equations:

$$
\begin{cases}u_{t}-\varepsilon \Delta u_{t}-\gamma \Delta u=f(x, y, t), & (x, y, t) \in \Omega \times[0, T], \\ u(x, y, t)=\varphi(x, y, t), & (x, y, t) \in \partial \Omega \times[0, T], \\ u(x, y, 0)=u_{0}(x, y), & (x, y) \in \Omega,\end{cases}
$$

where $u_{t}=\partial u / \partial t, \Delta u=\partial^{2} u / \partial x^{2}+\partial^{2} u / \partial y^{2}, \Delta u_{t}=\partial^{2} u_{t} / \partial x^{2}+\partial^{2} u_{t} / \partial y^{2}, T$ is the total time, $\varepsilon$ and $\gamma$ are two given positive constants, $f(x, y, t)$ is given source term, $u_{0}(x, y)$ and $\varphi(x, y, t)$ are initial and boundary value functions, respectively. For the sake of convenience, but without loss of generality, we assume $\varphi(x, y, t)=0$ in the following analysis.

The Sobolev equations hold very significant physical background so that they have become a class of important evolution partial differential equations (PDEs) and have been successfully used to many numerical simulations in mathematical and physical problems, such as the exchange in different media (see [1]) and the moisture migration in soil (see [2]). In particular, the Sobolev equations can be used to depict the porous phenomena saturated into rocks with cracks (see [3, 4]). However, the Sobolev equations in the real-

(c) The Author(s) 2018. This article is distributed under the terms of the Creative Commons Attribution 4.0 International License (http://creativecommons.org/licenses/by/4.0/), which permits unrestricted use, distribution, and reproduction in any medium, provided you give appropriate credit to the original author(s) and the source, provide a link to the Creative Commons license, and indicate if changes were made. 
world problems usually include the complex known data, such as the complicated initial and boundary value conditions, or the intricate source term, or the discontinuous coefficients, so that they have no analytic solution. Thus, they have to depend on numerical solutions.

At present, finite difference scheme (FDS), finite element method (FEM), finite volume element method (FVEM), and spectral method are considered to be four well-known numerical methods. By comparison, the spectral method can attain higher accuracy because it adopts Fourier and orthogonal polynomials to approximate unknown function, but other numerical methods use ordinary polynomials or difference quotient. Especially, with the rapid development of the electronic computers, the spectral method has achieved great success in many applied fields (see, e.g., $[5,6])$. The spectral method is a weighted residual way for PDEs and is usually classified as the Galerkin spectral method, the collocation spectral method (CSM), and the spectral element method, which are used to solve the second-order elliptic, parabolic, hyperbolic, hydromechanics PDEs, and so on (see [410]).

Although FDS, FEM, and FVEM have been used to solve the Sobolev equations (see $[3,4,10-13])$, the spectral method, especially CSM, has yet not been used to solve the 2D Sobolev equations, except a Fourier spectral method has been used to solve the onedimensional Sobolev equations in [14]. Therefore, in this article, we first develop a CSM for the 2D Sobolev equations. And then, we provide the existence, uniqueness, stability, and convergence for its CSM solutions. Finally, we give some numerical experiments to verify the correctness of theoretical results. Moreover, it is shown that the CSM scheme is very effective for solving the $2 \mathrm{D}$ Sobolev equations.

The rest of this article is arranged as follows. In Sect. 2, we first review the basic theory of spectral-collocation method and some Sobolev spaces. And then, in Sect. 3, we build the CSM scheme for the 2D Sobolev equations and analyze the existence, uniqueness, stability, and convergence of the CSM solutions. Next, in Sect. 4, we use some numerical experiments to verify that the results of numerical computations accord with the theoretical analysis and to certify that the CSM scheme is very efficient for solving the 2D Sobolev equations. Finally, we offer the main conclusions and discussion in Sect. 5.

\section{The basic theory of CSM and some Sobolev spaces}

\subsection{The basic theory of spectral-collocation method}

The rationale of the spectral methods is to approximate the solution $u$ of PDE with a finite sum $u_{N}$. In CSM, the approximate function $u_{N} \in P_{N}$ is denoted by its values at the Gauss-type interpolation points. In this study, we shall adopt the more common points, i.e., the so-called Chebyshev-Gauss-Lobatto (CGL) points (see [6]), as the interpolation nodes.

The Chebyshev polynomials are some special Jacobi polynomials, which are orthogonal with the Chebyshev weight function $\omega(x)=1 / \sqrt{1-x^{2}}$ over $[-1,1]$, namely

$$
\int_{-1}^{1} T_{m}(x) T_{n}(x) \omega(x) \mathrm{d} x=\gamma_{n} \delta_{m, n}
$$

where $\gamma_{n}=\left\|T_{n}\right\|_{\omega}^{2}=\int_{-1}^{1} T_{n}^{2}(x) \omega(x) \mathrm{d} x$. 
Let $\left\{x_{j}\right\}_{j=0}^{N}$ and $\left\{y_{k}\right\}_{k=0}^{N}$ be two sets of space nodes, i.e., the CGL points in $x$ and $y$ directions, respectively, and $\left\{\omega_{k}\right\}_{k=0}^{N}$ be a set of weights. Then they are, respectively, defined by

$$
x_{k}=-\cos \frac{\pi k}{N}, \quad y_{k}=-\cos \frac{k \pi}{N}, \quad \omega_{k}=\frac{\pi}{c_{k} N}, \quad 0 \leq k \leq N
$$

where $c_{0}=c_{N}=2$ and $c_{k}=1(k=1,2, \ldots, N-1)$. They hold the following property (see, e.g., [5]).

Theorem 1 Let $\left\{x_{k}\right\}_{k=0}^{N},\left\{y_{k}\right\}_{k=0}^{N}$, and $\left\{\omega_{k}\right\}_{k=0}^{N}$ be the sets of CGL quadrature nodes and weights, respectively. Then there holds

$$
\int_{-1}^{1} \int_{-1}^{1} p(x, y) \omega(x) \omega(y) \mathrm{d} x \mathrm{~d} y=\sum_{j=0}^{N} \sum_{k=0}^{N} p\left(x_{j}, y_{k}\right) \omega_{j} \omega_{k}, \quad \forall p(x, y) \in P_{2 N-1} .
$$

The fundamental of CSM is to get an approximation solution for $u(x, y)$ by a sum

$$
u_{N}(x, y)=\sum_{j=0}^{N} \sum_{k=0}^{N} u_{N}\left(x_{j}, y_{k}\right) h_{j}(x) h_{k}(y)
$$

where $u_{N}(x, y) \in P_{N}$, the interpolation nodes $\left\{x_{j}\right\}_{j=0}^{N}$ and $\left\{y_{k}\right\}_{k=0}^{N}$ are the CGL points given by (2), and $\left\{h_{j}(x)\right\}_{j=0}^{N}$ and $\left\{h_{k}(y)\right\}_{j=0}^{N}$ are the Lagrange basis polynomials associated with the sets of the CGL points $\left\{x_{j}\right\}_{j=0}^{N}$ and $\left\{y_{k}\right\}_{k=0}^{N}$, respectively.

Moreover, the derivative of $u_{N}(x, y)$ at $x_{k}$ is denoted by

$$
\frac{\partial u_{N}\left(x_{k}, y\right)}{\partial x}=\sum_{j=0}^{N} \sum_{l=0}^{N} u_{N}\left(x_{j}, y_{l}\right) h_{j}^{\prime}\left(x_{k}\right) h_{l}(y), \quad 0 \leq k \leq N .
$$

The first-order derivative of $h_{j}(x)$ at the CGL points can be denoted by the following explicit formulas:

$$
h_{j}^{\prime}\left(x_{k}\right)= \begin{cases}-\frac{2 N^{2}+1}{6}, & k=j=0, \\ \frac{c_{k}}{c_{j}} \frac{(-1)^{k+j}}{x_{k}-x_{j}}, & k \neq j, 0 \leq k, j \leq N, \\ -\frac{x_{k}}{2\left(1-x_{k}^{2}\right)}, & 1 \leq k=j \leq N-1, \\ \frac{2 N^{2}+1}{6}, & k=j=N,\end{cases}
$$

where $c_{0}=c_{N}=2$ and $c_{k}=1(k=1,2, \ldots, N-1)$. By replacing $x$ in (5) and (6) with $y$, we easily obtain the computational approach of $\partial u_{N}\left(x, y_{k}\right) / \partial y$.

\subsubsection{Some useful Sobolev spaces}

First, we provide some useful Sobolev spaces, whose detailed descriptions can be found in relative Reference [15]. 
Let $\Omega \in \mathbb{R}^{n}(n=1,2)$ be a bounded open domain with boundary $\partial \Omega$, and let $L^{2}(\Omega)$ denote the set of all square-integrable functions defined on $\Omega$.

For a non-negative integer $m$, and $\alpha=\left(\alpha_{1}, \alpha_{2}, \ldots, \alpha_{n}\right)\left(\alpha_{i} \geq 0\right.$ are integer and $|\alpha|=$ $\left.\sum_{i=1}^{n} \alpha_{i}\right)$, define

$$
H^{m}(\Omega)=\left\{u \in L^{2}(\Omega): D^{\alpha} u \in L^{2}(\Omega), 0 \leq|\alpha| \leq m\right\},
$$

equipped with the norm and semi-norm as follows, respectively:

$$
\|u\|_{m}=\left(\sum_{0 \leq|\alpha| \leq m}\left\|D^{\alpha} u\right\|_{0}^{2}\right)^{1 / 2}, \quad|u|_{m}=\left(\sum_{|\alpha|=m}\left\|D^{\alpha} u\right\|_{0}^{2}\right)^{1 / 2} .
$$

Set $H_{0}^{m}(\Omega)=\left\{u \in H^{m}(\Omega):\left.D^{\alpha} u(x)\right|_{\partial \Omega}=0,|\alpha|<m\right\}$ and $H^{-m}(\Omega)$ denotes the dual space of $H_{0}^{m}(\Omega)$.

Further, let $\omega=: \omega(x, y)=\omega(x) \omega(y)=1 / \sqrt{\left(1-x^{2}\right)\left(1-y^{2}\right)}, \Omega=(-1,1)^{2}, L_{\omega}^{2}(\Omega)$ denote the set of all square-integrable functions defined on $\Omega$, equipped with the norm

$$
\|u\|_{0, \omega}=\left(\int_{\Omega}|u|^{2} \omega \mathrm{d} \Omega\right)^{1 / 2}
$$

and $H_{\omega}^{m}(\Omega):=\left\{u \in L_{\omega}^{2}(\Omega): D^{\alpha} u \in L_{\omega}^{2}(\Omega), 0 \leq|\alpha| \leq m\right\}$ be a weighted Sobolev space on $\Omega$ with the CGL quadrature weight function, equipped with the norm

$$
\|u\|_{m, \omega}=\left(\sum_{0 \leq|\alpha| \leq m}\left\|D^{\alpha} u\right\|_{0, \omega}^{2}\right)^{\frac{1}{2}}, \quad\|u\|_{0, \omega}=\left(\int_{\Omega}|u|^{2} \omega \mathrm{d} \Omega\right)^{\frac{1}{2}}, \quad \omega=\omega(x) \omega(y) .
$$

Furthermore, set $H_{0, \omega}^{1}(\Omega)=\left\{u \in H_{\omega}^{1}(\Omega):\left.u\right|_{\partial \Omega}=0\right\},(\cdot, \cdot)_{\omega}$ denotes the weighted inter product of $L_{\omega}^{2}(\Omega)=H_{\omega}^{0}(\Omega)$, and $\|\cdot\|_{H^{l}\left(H_{\omega}^{m}\right)}$ is the norm in the following space:

$$
H^{l}\left(0, T ; H_{\omega}^{m}(\Omega)\right) \equiv\left\{v(t) \in H_{\omega}^{m}(\Omega):\|v\|_{H^{l}\left(H_{\omega}^{m}\right)}^{2} \equiv \int_{0}^{T} \sum_{i=0}^{l}\left\|\frac{\mathrm{d}^{i}}{\mathrm{~d} t^{i}} v(t)\right\|_{m, \omega}^{2} \mathrm{~d} t<\infty\right\} .
$$

Next, define the $H_{\omega}^{1}$-orthogonal projection $R_{N}: H_{0, \omega}^{1}(\Omega) \rightarrow P_{N}$ such that, for any $u \in$ $H_{0, \omega}^{1}(\Omega)$, there holds

$$
\left(\nabla\left(R_{N} u-u\right), \nabla v\right)_{\omega}=0, \quad \forall v \in P_{N},
$$

where $\Omega=[-1,1]^{2}$ and $(\cdot, \cdot)_{\omega}$ is the inner product in $L_{\omega}^{2}(\Omega)^{2}$ about the Chebyshev weight function $\omega=\omega(x, y)=\omega(x) \omega(y)=1 / \sqrt{\left(1-x^{2}\right)\left(1-y^{2}\right)}$, or equivalently,

$$
u_{N}(x, y)=R_{N} u(x, y)=\sum_{j=0}^{N} \sum_{k=0}^{N} u_{N}\left(x_{j}, y_{k}\right) h_{j}(x) h_{k}(y)
$$

Therefore, we can also approximate the unknown solution $u(x, y)$ with $R_{N} u(x, y)$. In addition, $R_{N}$ has the following important property (see [6, Chapter III]). 
Theorem 2 For any $u \in H_{\omega}^{q}(\Omega)$ with $q \geq 2$, we have

$$
\left\|\nabla R_{N} u\right\|_{0, \omega} \leq\|\nabla u\|_{0, \omega}, \quad\left\|\partial^{k}\left(R_{N} u-u\right)\right\|_{0, \omega}=O\left(N^{k-q}\right), \quad 0 \leq k \leq q \leq N+1 .
$$

Finally, we provide several formulas used often in the following discussions.

(1) The Poincaré inequality

There exists a constant $C_{p}$ such that

$$
C_{p}\|u\|_{m} \leq|u|_{m} \leq\|u\|_{m}, \quad \forall u \in H_{0}^{m}(\Omega)
$$

(2) The Hölder inequality

$$
\int_{\Omega}|u v| \mathrm{d} \Omega \leq\left(\int_{\Omega}|u|^{2} \mathrm{~d} \Omega\right)^{\frac{1}{2}}\left(\int_{\Omega}|v|^{2} \mathrm{~d} \Omega\right)^{\frac{1}{2}}, \quad \forall u, v \in L^{2}(\Omega) .
$$

(3) Green's formula

$$
\int_{\Omega} v \Delta u \mathrm{~d} \Omega=-\int_{\Omega} \nabla u \cdot \nabla v \mathrm{~d} \Omega+\int_{\partial \Omega} v \frac{\partial u}{\partial \mathbf{n}} \mathrm{d} s, \quad \forall u \in H^{2}(\Omega), \forall v \in H^{1}(\Omega),
$$

where $\Delta u=\sum_{i=1}^{n} \partial^{2} u / \partial x_{i}^{2}, \nabla u=\left(\partial u / \partial x_{1}, \partial u / \partial x_{2}, \ldots, \partial u / \partial x_{n}\right)$, and $\mathbf{n}$ is the unit outer normal vector on $\partial \Omega$.

(4) The Cauchy inequality

$$
a b \leq \frac{\varepsilon a^{2}}{2}+\frac{b^{2}}{2 \varepsilon}, \quad \forall a \geq 0, b \geq 0, \varepsilon>0 .
$$

\section{CSM for the 2D Sobolev equations}

\subsection{The variational formulation for the $2 \mathrm{D}$ Sobolev equations}

By Green's formula, we can attain the following variational formulation for the 2D Sobolev equations (1).

Problem 3 For $t \in(0, T)$, find $u \in H_{0, \omega}^{1}(\Omega)$ such that

$$
\left\{\begin{array}{l}
\left(u_{t}, v\right)_{\omega}+\varepsilon\left(\nabla u_{t}, \nabla v\right)_{\omega}+\gamma(\nabla u, \nabla v)_{\omega}=(f, v)_{\omega}, \quad \forall v \in H_{0, \omega}^{1}(\Omega) \\
u(x, y, 0)=u_{0}(x, y), \quad(x, y) \in \Omega
\end{array}\right.
$$

For Problem 3, we have the following result of the existence, uniqueness, and stability of the generalized solution.

Theorem 4 Iff $\in L^{2}\left(0, T ; L_{\omega}^{2}(\Omega)\right)$ and $u_{0} \in H_{\omega}^{1}(\Omega)$, then there exists a unique generalized solution for the variational formulation (8) satisfying the following stability:

$$
\|u\|_{1, \omega} \leq \tilde{c}\left(\left\|u_{0}\right\|_{1, \omega}+\|f\|_{L^{2}\left(L_{\omega}^{2}\right)}\right)
$$

where $\tilde{c}=\sqrt{\max \left\{1, \varepsilon, 1 /\left(\gamma C_{p}^{2}\right)\right\} / \min \{1, \varepsilon\}}$. 
Proof Because (8) is a system of linear equations about unknown function $u$, to demonstrate that there exists a unique solution for the variational formulation (8) is equivalent to proving that equation (8) has only a zero solution when $f(x, y, t)=u_{0}(x, y)=0$.

Taking $v=u$ in the first formula of equation (8), we have

$$
\frac{\mathrm{d}\|u\|_{0, \omega}^{2}}{2 \mathrm{~d} t}+\varepsilon \frac{\mathrm{d}\|\nabla u\|_{0, \omega}^{2}}{2 \mathrm{~d} t}+\gamma\|\nabla u\|_{0, \omega}^{2}=(f, u)_{\omega}
$$

By integrating (10) from 0 to $t \in[0, T]$ and by the Hölder, Poincaré, and Cauchy inequalities, we obtain

$$
\begin{aligned}
& \|u\|_{0, \omega}^{2}+\varepsilon\|\nabla u\|_{0, \omega}^{2}+2 \gamma \int_{0}^{t}\|\nabla u\|_{0, \omega}^{2} d t \\
& \quad=\left\|u_{0}\right\|_{0, \omega}^{2}+\varepsilon\left\|\nabla u_{0}\right\|_{0, \omega}^{2}+2 \int_{0}^{t}(f, u)_{\omega} \mathrm{d} t \\
& \quad \leq\left\|u_{0}\right\|_{0, \omega}^{2}+\varepsilon\left\|\nabla u_{0}\right\|_{0, \omega}^{2}+\frac{1}{\gamma C_{p}^{2}} \int_{0}^{t}\|f\|_{0, \omega}^{2} \mathrm{~d} t+\gamma \int_{0}^{t}\|\nabla u\|_{0, \omega}^{2} \mathrm{~d} t .
\end{aligned}
$$

Therefore, when $f(x, y, t)=u_{0}(x, y)=0$, we obtain $\|u\|_{0, \omega}=\|\nabla u\|_{0, \omega}=0$, which implies $u=$ 0 , namely the variational formulation (8) has a unique solution $u \in H_{0, \omega}^{1}(\Omega)$. Further, from (11), we obtain (9). This completes the proof of Theorem 4.

\subsection{CSM for the 2D Sobolev equations}

When solving time-dependent PDEs by CSM, we use FDS in time and the spectral method in space. In the following discussions, for convenience, we can assume $a=c=-1$ and $b=d=1$ because, by employing transforms $x^{\prime}=-1+2(x-a) /(b-a)$ and $y^{\prime}=-1+$ $2(y-c) /(d-c)$, we can ensure $[a, b] \leftrightarrow[-1,1]$ and $[c, d] \leftrightarrow[-1,1]$, respectively.

\subsubsection{Establishment of the CSM scheme}

The main idea of CSM is to seek an approximate solution at time and spatial nodes. In this article, we will take the CGL type interpolation points as the space nodes. Namely, let $\left\{x_{j}\right\}_{j=0}^{N}$ and $\left\{y_{k}\right\}_{k=0}^{N}$ be the space nodes in $x$ and $y$ directions, respectively, with

$$
x_{j}=-\cos \frac{j \pi}{N}, \quad y_{k}=-\cos \frac{k \pi}{N},
$$

where the positive integer $N$ denotes the number of nodes in a certain direction. And, for integer $K>0$, let $\Delta t=T / K$ be the time step, i.e., $K \Delta t=T$. We approximate $u(x, y, n \Delta t)$ with $u^{n}$, the time derivative $u_{t}$ of $u(x, y, t)$ at time $t_{n}=n \Delta t$ with $\left(u^{n+1}-u^{n}\right) / \Delta t$, and $u^{n}(x, y)$ with $u_{N}^{n}(x, y)$, namely

$$
u^{n}(x, y) \approx u_{N}^{n}(x, y)=\sum_{j=0}^{N} \sum_{k=0}^{N} u_{N}^{n}\left(x_{j}, y_{k}\right) h_{j}(x) h_{k}(y), \quad 0 \leq n \leq K .
$$

Then, we obtain the following CSM scheme for the 2D Sobolev equations. 
Problem 5 Find $u_{N}^{n} \in U_{N} \equiv H_{0, \omega}^{1}(\Omega) \cap P_{N}$ such that

$$
\left\{\begin{aligned}
\left(u_{N}^{n+1}-u_{N}^{n}, v_{N}\right)_{\omega}+\varepsilon\left(\nabla u_{N}^{n+1}-\nabla u_{N}^{n}, \nabla v_{N}\right)_{\omega}+\gamma \Delta t\left(\nabla u_{N}^{n+1}, \nabla v_{N}\right)_{\omega} \\
=\Delta t\left(f\left(t_{n+1}\right), v_{N}\right)_{\omega}, \quad \forall v_{N} \in U_{N}, 0 \leq n \leq K, \\
u_{N}^{0}(x, y)=R_{N} u_{0}(x, y), \quad(x, y) \in \Omega,
\end{aligned}\right.
$$

where $f\left(t_{n}\right)=f\left(x, y, t_{n}\right)$.

\subsubsection{Existence, uniqueness, and stability of the CSM solutions}

For Problem 5, we have the result of the existence, uniqueness, and stability about the CSM solutions.

Theorem 6 Iff $\in L^{2}\left(0, T ; L_{\omega}^{2}(\Omega)\right)$ and $u_{0} \in H_{\omega}^{1}(\Omega)$, then there exists a unique series of solutions $u_{N}^{n} \in U_{N}(n=1,2, \ldots, K)$ for the CSM scheme (12) satisfying the following stability:

$$
\left\|\nabla u_{N}^{n}\right\|_{0, \omega} \leq\left\|\nabla u_{0}\right\|_{0, \omega}+\left[\frac{\Delta t}{\gamma} \sum_{j=1}^{n}\left\|f\left(t_{j}\right)\right\|_{0, \omega}^{2}\right]^{1 / 2}, \quad n=1,2, \ldots, K .
$$

Proof Because scheme (12) is a linear system of equations about $u_{N}^{n+1}$, in order to demonstrate the existence and uniqueness of solutions for the CSM scheme (12), it is necessary to prove that (12) has only zero solution when $u_{0}(x, y)=f(x, y, t)=0$.

By taking $v_{N}=u_{N}^{n+1}-u_{N}^{n}$ in the first equation of (12), we have

$$
\begin{gathered}
\left\|u_{N}^{n+1}-u_{N}^{n}\right\|_{0, \omega}^{2}+\varepsilon\left\|\nabla u_{N}^{n+1}-\nabla u_{N}^{n}\right\|_{0, \omega}^{2}+\gamma \Delta t\left\|\nabla u_{N}^{n+1}\right\|_{0, \omega}^{2} \\
=\gamma \Delta t\left(\nabla u_{N}^{n+1}, \nabla u_{N}^{n}\right)_{0, \omega}+\Delta t\left(f\left(t_{n+1}\right), u_{N}^{n+1}-u_{N}^{n}\right)_{0, \omega} .
\end{gathered}
$$

Then, by the Hölder and Cauchy inequalities, we obtain

$$
\begin{aligned}
& \left\|u_{N}^{n+1}-u_{N}^{n}\right\|_{0, \omega}^{2}+\varepsilon\left\|\nabla u_{N}^{n+1}-\nabla u_{N}^{n}\right\|_{0, \omega}^{2}+\gamma \Delta t\left\|\nabla u_{N}^{n+1}\right\|_{0, \omega}^{2} \\
& \quad \leq \frac{\gamma \Delta t}{2}\left\|\nabla u_{N}^{n+1}\right\|_{0, \omega}^{2}+\frac{\gamma \Delta t}{2}\left\|\nabla u_{N}^{n}\right\|_{0, \omega}^{2}+\frac{\Delta t^{2}}{2}\left\|f\left(t_{n+1}\right)\right\|_{0, \omega}^{2}+\frac{1}{2}\left\|u_{N}^{n+1}-u_{N}^{n}\right\|_{0, \omega}^{2} .
\end{aligned}
$$

By summing (15) from 1 to $n$ and using the second formula of (12), we obtain

$$
\begin{gathered}
\sum_{j=0}^{n}\left\|u_{N}^{j+1}-u_{N}^{j}\right\|_{0, \omega}^{2}+2 \varepsilon \sum_{j=0}^{n}\left\|\nabla u_{N}^{j+1}-\nabla u_{N}^{j}\right\|_{0, \omega}^{2}+\gamma \Delta t\left\|\nabla u_{N}^{n+1}\right\|_{0, \omega}^{2} \\
\leq \gamma \Delta t\left\|\nabla u_{0}\right\|_{0, \omega}^{2}+\Delta t^{2} \sum_{j=0}^{n}\left\|f\left(t_{j+1}\right)\right\|_{0, \omega}^{2}, \quad n=0,1,2, \ldots, K-1 .
\end{gathered}
$$

Thus, from (16), we obtain $\left\|\nabla u_{N}^{n+1}\right\|_{\omega}=0(n=0,1, \ldots, K-1)$ when $f=u_{0}=0$, which implies $u_{N}^{n}=0(n=1,2, \ldots, K)$. In other words, the CSM scheme (12) has a unique series of solutions. From (16), we immediately attain (13). This completes the proof of Theorem 6. 


\subsubsection{The convergence of the CSM solutions}

For the series of solutions of Problem 5, we have the following conclusion of convergence.

Theorem 7 Under the same conditions of Theorem 6, if the solutions of Problem $3 u\left(t_{n}\right) \in$ $H_{\omega}^{q}(\Omega)(2 \leq q \leq N+1)$, when $\Delta t=O\left(N^{-1}\right)$, the errors between the solution for Problem 3 and the series of solutions of Problem 5 have the following estimates:

$$
\left\|\nabla\left(u\left(t_{n}\right)-u_{N}^{n}\right)\right\|_{0, \omega}=O\left(\Delta t, N^{1-q}\right), \quad 1 \leq n \leq K, 2 \leq q \leq N+1
$$

Proof Let $e_{1}^{n}=u\left(t_{n}\right)-u^{n}, e_{2}^{n}=u^{n}-R_{N} u^{n}$, and $e_{3}^{n}=R_{N} u^{n}-u_{N}^{n}$.

(1) First, estimate $e_{1}^{n}$.

If we adopt $\left(u^{n+1}-u^{n}\right) / \Delta t$ to approximate $u_{t}$, we obtain the following semi-discretize formulation of equation (8) about time:

$$
\begin{aligned}
& \frac{1}{\Delta t}\left(u^{n+1}-u^{n}, v\right)_{\omega}+\frac{\varepsilon}{\Delta t}\left(\nabla u^{n+1}-\nabla u^{n}, \nabla v\right)_{\omega}+\gamma\left(\nabla u^{n+1}, \nabla v\right)_{\omega} \\
& \quad=\left(f\left(t_{n+1}\right), v\right)_{\omega}, \quad \forall v \in H_{0, \omega}^{1}(\Omega) .
\end{aligned}
$$

At time $t=t_{n}$, by applying Taylor's expansion to (8) and subtracting (18), taking $v=e_{1}^{n+1}-$ $e_{1}^{n}$, we obtain

$$
\begin{aligned}
\left\|e_{1}^{n+1}-e_{1}^{n}\right\|_{0, \omega}^{2}+\varepsilon\left\|\nabla\left(e_{1}^{n+1}-e_{1}^{n}\right)\right\|_{0, \omega}^{2}+\gamma \Delta t\left\|\nabla e_{1}^{n+1}\right\|_{0, \omega}^{2} \\
=\frac{\Delta t^{2}}{2}\left(u_{t t}\left(\xi_{1}^{n}\right), e_{1}^{n+1}-e_{1}^{n}\right)_{\omega} \\
\quad+\frac{\varepsilon \Delta t^{2}}{2}\left(\nabla u_{t t}\left(\xi_{2}^{n}\right), \nabla\left(e_{1}^{n+1}-e_{1}^{n}\right)\right)_{\omega} \\
\quad+\gamma \Delta t\left(\nabla e_{1}^{n+1}, \nabla e_{1}^{n}\right)_{\omega}
\end{aligned}
$$

where $t_{n} \leq \xi_{1}^{n}, \xi_{2}^{n} \leq t_{n+1}$. By using the Hölder and Cauchy inequalities, we obtain

$$
\begin{aligned}
\left\|e_{1}^{n+1}-e_{1}^{n}\right\|_{0, \omega}^{2}+\varepsilon\left\|\nabla\left(e_{1}^{n+1}-e_{1}^{n}\right)\right\|_{0, \omega}^{2}+\gamma \Delta t\left\|\nabla e_{1}^{n+1}\right\|_{0, \omega}^{2} \\
\leq \frac{1}{2}\left(\frac{\Delta t^{2}}{2}\right)^{2}\left\|u_{t t}\left(\xi_{1}^{n}\right)\right\|_{0, \omega}^{2}+\frac{1}{2}\left\|e_{1}^{n+1}-e_{1}^{n}\right\|_{0, \omega}^{2} \\
\quad+\frac{\varepsilon}{2}\left(\frac{\Delta t^{2}}{2}\right)^{2}\left\|\nabla u_{t t}\left(\xi_{2}^{n}\right)\right\|_{0, \omega}^{2} \\
\quad+\frac{\varepsilon}{2}\left\|\nabla\left(e_{1}^{n+1}-e_{1}^{n}\right)\right\|_{0, \omega}^{2}+\frac{\gamma \Delta t}{2}\left(\left\|\nabla e_{1}^{n+1}\right\|_{0, \omega}^{2}+\left\|\nabla e_{1}^{n}\right\|_{0, \omega}^{2}\right) .
\end{aligned}
$$

Further, we obtain

$$
\begin{aligned}
\gamma \Delta t\left\|\nabla e_{1}^{n+1}\right\|_{0, \omega}^{2} \leq & \frac{\Delta t^{4}}{4}\left\|u_{t}\left(\xi_{1}^{n}\right)\right\|_{0, \omega}^{2}+\frac{\varepsilon \Delta t^{4}}{4}\left\|\nabla u_{t t}\left(\xi_{2}^{n}\right)\right\|_{0, \omega}^{2} \\
& +\gamma \Delta t\left\|\nabla e_{1}^{n}\right\|_{0, \omega}^{2}
\end{aligned}
$$


As $e_{1}^{0}=0$, by summing (21) from 0 to $n$, we obtain

$$
\left\|\nabla e_{1}^{n+1}\right\|_{0, \omega}^{2} \leq \frac{\Delta t^{3}}{4 \gamma} \sum_{j=0}^{n}\left(\left\|u_{t}\left(\xi_{1}^{j}\right)\right\|_{0, \omega}^{2}+\varepsilon\left\|\nabla u_{t t}\left(\xi_{2}^{j}\right)\right\|_{0, \omega}^{2}\right)
$$

namely

$$
\left\|\nabla e_{1}^{n+1}\right\|_{0, \omega} \leq C \Delta t, \quad 0 \leq n \leq K-1
$$

where $C^{2}=\frac{\Delta t}{4 \gamma} \sum_{j=0}^{n}\left(\left\|u_{t t}\left(\xi_{1}^{n}\right)\right\|_{0, \omega}^{2}+\varepsilon\left\|u_{t t}\left(\xi_{2}^{n}\right)\right\|_{0, \omega}^{2}\right)$.

(2) Next, estimate $e_{2}$.

The estimate of $e_{2}$ can be immediately obtained by Theorem 2, i.e.,

$$
\left\|\nabla e_{2}^{n}\right\|_{0, \omega}=O\left(N^{1-q}\right), \quad n=1,2, \ldots, K, 2 \leq q \leq N+1
$$

(3) Finally, discuss the estimate of $e_{3}=R_{N} u^{n}-u_{N}^{n}$.

By subtracting Problem 5 from (18) taking $v=v_{N} \in U_{N}$, we obtain

$$
\begin{gathered}
\left(u^{n+1}-u_{N}^{n+1}-\left(u^{n}-u_{N}^{n}\right), v_{N}\right)_{\omega}+\gamma \Delta t\left(\nabla\left(u^{n+1}-u_{N}^{n+1}\right), \nabla v_{N}\right)_{\omega} \\
+\varepsilon\left(\nabla\left(u^{n+1}-u_{N}^{n+1}-\left(u^{n}-u_{N}^{n}\right)\right), \nabla v_{N}\right)_{\omega}=0, \quad \forall v_{N} \in U_{N} .
\end{gathered}
$$

By Theorem 2, (24), the property of $R_{N}$, and the Hölder and Cauchy inequalities, we have

$$
\begin{aligned}
\| e_{3}^{n+1} & -e_{3}^{n}\left\|_{0, \omega}^{2}+\varepsilon\right\| \nabla\left(e_{3}^{n+1}-e_{3}^{n}\right)\left\|_{0, \omega}^{2}+\gamma \Delta t\right\| \nabla e_{3}^{n+1} \|_{0, \omega}^{2} \\
= & \left(u^{n+1}-u^{n}-\left(u_{N}^{n+1}-u_{N}^{n}\right), e_{3}^{n+1}-e_{3}^{n}\right) \\
& +\left(R_{N} u^{n+1}-u^{n+1}-\left(R_{N} u^{n}-u^{n}\right), e_{3}^{n+1}-e_{3}^{n}\right) \\
& +\varepsilon\left(\nabla\left(u^{n+1}-u_{N}^{n+1}-\left(u^{n}-u_{N}^{n}\right)\right), \nabla\left(e_{3}^{n+1}-e_{3}^{n}\right)\right) \\
& +\varepsilon\left(\nabla\left(R_{N} u^{n+1}-u^{n+1}-\left(R_{N} u^{n}-u^{n}\right)\right), \nabla\left(e_{3}^{n+1}-e_{3}^{n}\right)\right) \\
& +\gamma \Delta t\left(\nabla\left(R_{N} u^{n+1}-u^{n+1}\right), \nabla\left(e_{3}^{n+1}-e_{3}^{n}\right)\right) \\
& +\gamma \Delta t\left(\nabla\left(u^{n+1}-u_{N}^{n+1}\right), \nabla\left(e_{3}^{n+1}-e_{3}^{n}\right)\right) \\
= & \left(R_{N} u^{n+1}-u^{n+1}-\left(R_{N} u^{n}-u^{n}\right), e_{3}^{n+1}-e_{3}^{n}\right) \\
\leq & \frac{1}{2}\left\|e_{3}^{n+1}-e_{3}^{n}\right\|_{0, \omega}^{2}+\left\|R_{N} u^{n+1}-u^{n+1}\right\|_{0, \omega}^{2}+\left\|R_{N} u^{n}-u^{n}\right\|_{0, \omega}^{2} \\
\leq & \frac{1}{2}\left\|e_{3}^{n+1}-e_{3}^{n}\right\|_{0, \omega}^{2}+C N^{-2 q}, \quad n=0,1, \ldots, K-1,2 \leq q \leq N+1 .
\end{aligned}
$$

When $\Delta t=O\left(N^{-1}\right)$, from (25), we attain

$$
\left\|\nabla e_{3}^{n}\right\|_{0, \omega}=O\left(N^{-q-1 / 2}\right), \quad n=1,2, \ldots, K, 2 \leq q \leq N+1
$$

By combining (22)-(23) with (26), we attain (17). This completes the proof of Theorem 7. 
By using the Nietzsche technique and Theorem 7, we easily obtain the following $L_{\omega}^{2}$ norm error estimates.

Corollary 8 Under the same conditions of Theorem 6, when $\Delta t=O\left(N^{-1}\right)$, the $L_{\omega}^{2}$ norm error estimates between the solution for Problem 3 and the series of solutions of Problem 5 are as follows:

$$
\left\|u\left(t_{n}\right)-u_{N}^{n}\right\|_{0, \omega}=O\left(\Delta t^{2}, N^{-q}\right), \quad 1 \leq n \leq K, 2 \leq q \leq N+1 .
$$

Remark 1 The error estimates in Theorem 7 and Corollary 8 attain an optimal order because one can only ensure $u \in H^{1}\left(0, T ; H_{\omega}^{2}(\Omega)\right)$ when $f \in L^{2}\left(0, T ; L_{\omega}^{2}(\Omega)\right)$ and $u_{0} \in H_{\omega}^{1}(\Omega)$. Theorem 6 shows that the CSM scheme, i.e., Problem 5 for the 2D Sobolev equations, has a unique series of solutions which is stable and continuously depends on the initial value and source functions. This theoretically ensures that Problem 5 is effective and reliable for solving the 2D Sobolev equations.

\section{Numerical experiments}

In this section, we give some numerical experiments to verify the correction of the theoretical results of the CSM scheme, i.e., Problem 5 for the $2 \mathrm{D}$ Sobolev equations.

In the $2 \mathrm{D}$ Sobolev equation (1), we took $\bar{\Omega}=[-1,1] \times[-1,1], \varepsilon=1 / \pi^{2}, \gamma=2 / \pi^{2}, \varphi=0$, $u_{0}(x, y)=1-\cos 2 \pi x \cos 2 \pi y$, and $f(x, y, t)=2(\cos 2 \pi x \cos 2 \pi y-1) \exp (-2 t)$. Thus, we can find the analytical solutions for the Sobolev equations (1) as follows:

$$
u(x, y, t)=(1-\cos 2 \pi x \cos 2 \pi y) \exp (-2 t), \quad(x, y, t) \in[-1,1] \times[-1,1] \times[0, \infty) .
$$

When we take time step $\Delta t=0.01$ and the number of nodes in every direction $N=$ 100 , from Corollary 8 , the theoretical errors between the analytical solution and the CSM solutions $u_{N}^{k}(k=1,2, \ldots, K)$ should be $O\left(10^{-4}\right)$.

By the CSM scheme (12), we obtained the CSM numerical solutions at $T=0,0.3,0.5$, 0.9 , depicted in (a)'s of Figs. 1 to 4, respectively. The analytical solutions at the same time nodes are depicted in (b)'s of Figs. 1 to 4, respectively. Each pair of photos in Figs. 1 to 4 are almost the same.

Photos (a), (b), and (c) in Fig. 5 show the errors between the analytical solutions and the CSM solutions when $t=0.3, t=0.5$, and $t=0.9$, respectively, which indicate that the

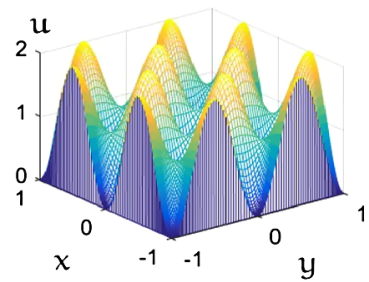

(a)

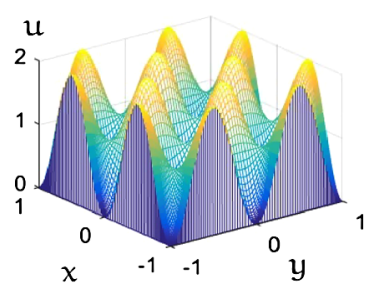

(b)

Figure 1 (a) The analytical solution when $t=0$. (b) The CSM solution when $t=0$ 


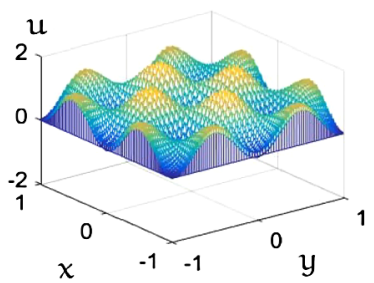

(a)

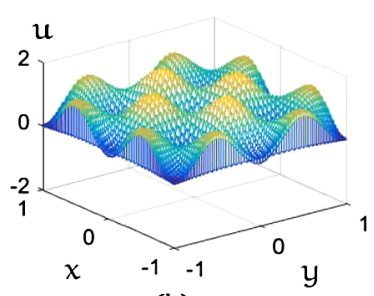

(b)

Figure 2 (a) The analytical solution when $t=0.3$. (b) The CSM solution when $t=0.3$

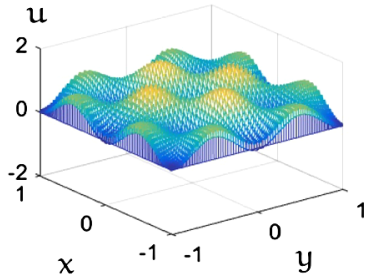

(a)

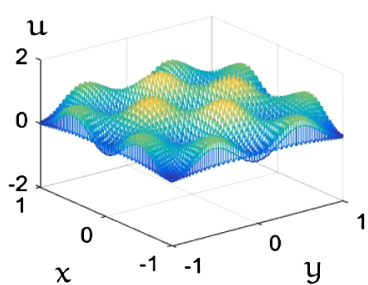

(b)

Figure 3 (a) The analytical solution when $t=0.5$. (b) The CSM solution when $t=0.5$

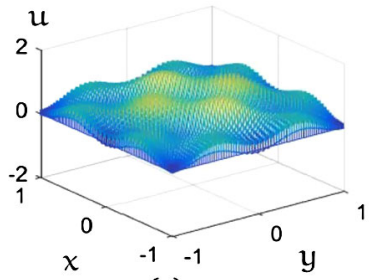

(a)

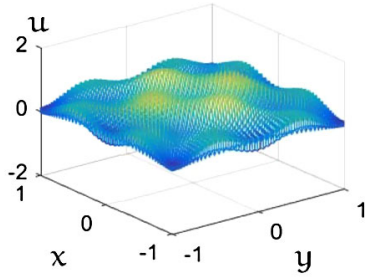

(b)

Figure 4 (a) The analytical solution when $t=0.9$. (b) The CSM solution when $t=0.9$

numerical computational errors accord with the theoretical ones, because both errors are $O\left(10^{-4}\right)$. This implies that the CSM scheme is very efficient and feasible for solving the $2 \mathrm{D}$ Sobolev equations (1).

\section{Conclusions and discussion}

In this work, we have established the CSM scheme by means of the Chebyshev polynomials for the 2D Sobolev equations, analyzed the the existence, uniqueness, stability, and convergence of the CSM solutions. We have also used some numerical experiments to check the feasibility and effectiveness of the CSM scheme and to verity that the numerical computing results accord with the theoretical analysis ones. Moreover, it is shown that the CSM scheme is very valid and feasible for solving the 2D Sobolev equations.

Even if we only study CSM for the 2D Sobolev equations, the CSM scheme can be easily and effectively used to solve for the Sobolev equations in a three-dimensional space or the Sobolev equations with complex geometric domains. 


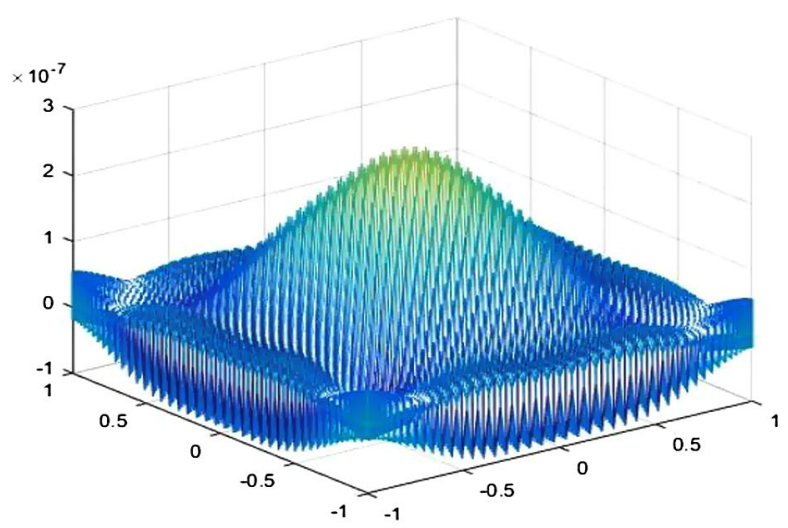

(a)

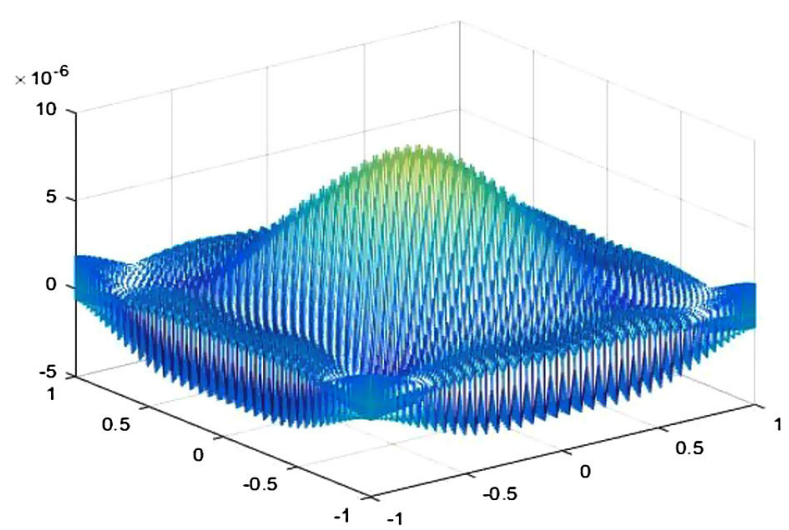

(b)

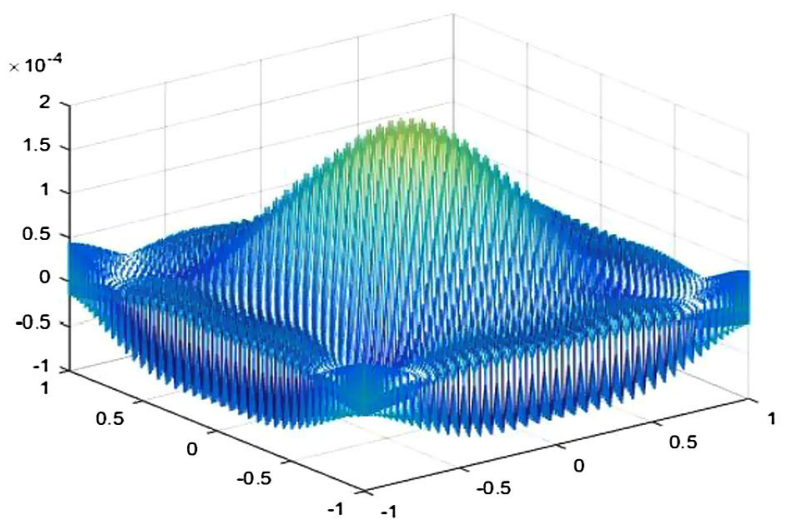

(c)

Figure 5 Errors between the analytical solutions and the CSM solutions: (a) at $t=0.3$, (b) at $t=0.5$, and (c) at $t=0.9$

\section{Acknowledgements}

The authors are thankful to the honorable reviewers and editors for their valuable suggestions and comments, which improved the paper.

\section{Funding}

This research was supported by the National Science Foundation of China grant 11671106. 
Competing interests

The authors declare that they have no competing interests.

\section{Authors' contributions}

All authors contributed equally and significantly in writing this article. All authors wrote, read, and approved the final manuscript.

\section{Author details}

${ }^{1}$ School of Control and Computer Engineering, North China Electric Power University, Beijing, China. ${ }^{2}$ School of Mathematics and Physics, North China Electric Power University, Beijing, China.

\section{Publisher's Note}

Springer Nature remains neutral with regard to jurisdictional claims in published maps and institutional affiliations.

Received: 30 January 2018 Accepted: 10 May 2018 Published online: 25 May 2018

\section{References}

1. Ting, T.W.: A cooling process according to two-temperature theory of heat conduction. J. Math. Anal. Appl. 45(1), 23-31 (1974)

2. Shi, D.M.: On the initial boundary value problem of nonlinear equation of the migration of the moisture in soil. Acta Math. Appl. Sin. 13(1), 31-38 (1990)

3. Liu, Y., Li, H., He, S., Gao, W., Mu, S.: A new mixed scheme based on variation of constants for Sobolev equation with nonlinear convection term. Appl. Math. J. Chin. Univ. 28(2), 158-172 (2013)

4. Shi, D.Y., Wang, H.H.: Nonconforming H 1-Galerkin mixed FEM for Sobolev equations on anisotropic meshes. Acta Math. Appl. Sin. 25(02), 335-344 (2009)

5. Guo, B.Y.: Spectral Methods and Their Applications. World Scientific, Singapore (1998)

6. Shen, J., Tang, T.: Spectral and High-Order Methods with Applications. Science Press, Beijing (2006)

7. Luo, Z.D., Jin, S.J.: A reduced-order extrapolation spectral-finite difference scheme based on the POD method for 2D second-order hyperbolic equations. Math. Model. Anal. 22(5), 569-586 (2017)

8. An, J., Luo, Z.D., Li, H., Sun, P.: Reduced-order extrapolation spectral-finite difference scheme based on POD method and error estimation for three-dimensional parabolic equation. Front. Math. China 10(5), 1025-1040 (2015)

9. Guo, B.Y.: Some progress in spectral methods. Sci. China Math. 56(12), 2411-2438 (2013)

10. Jiang, Z.W., Chen, H.Z.: Error estimates for mixed finite element methods for Sobolev equation. Northeast. Math. J. 17(3), 301-314 (2001)

11. Gao, F.Z., Qiu, J.X., Zhang, Q.: Local discontinuous Galerkin finite element method and error estimates for one class of Sobolev equation. J. Sci. Comput. 41, 436-460 (2009)

12. Shi, D.Y., Wang, H.H., Guo, C.: Anisotropic rectangular nonconforming finite element analysis for Sobolev equations. Appl. Math. Mech. 29(9), 1203-1214 (2008)

13. Li, H., Luo, Z.D., An, J.: A fully discrete finite volume element formulation for Sobolev equation and numerical simulations. Math. Numer. Sin. 34(2), 163-172 (2010)

14. Lu, W.J., Zhang, F.Y.: Long-time behavior of completely discrete Fourier spectral method of solutions to Sobolev equations. J. Natur. Sci. Heilongjiang Univ. 18(2), 5-8 (2001)

15. Adams, R.A.: Sobolev Spaces. Academic Press, New York (1975)

\section{Submit your manuscript to a SpringerOpen ${ }^{\circ}$ journal and benefit from:}

- Convenient online submission

- Rigorous peer review

- Open access: articles freely available online

- High visibility within the field

- Retaining the copyright to your article 